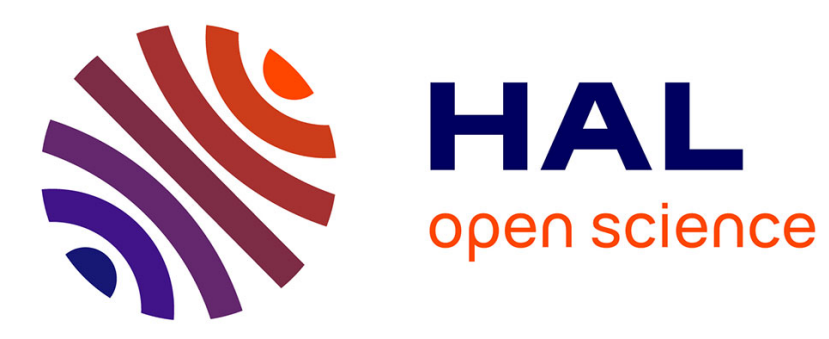

\title{
Charged particle dynamics in a tangential discontinuity
}

Roch Smets

\section{To cite this version:}

Roch Smets. Charged particle dynamics in a tangential discontinuity. Journal of Geophysical Research Space Physics, 2000, 105 (A11), pp.25009-25020. 10.1029/1999JA000173 . hal-02994646

\section{HAL Id: hal-02994646 \\ https://hal.science/hal-02994646}

Submitted on 8 Nov 2020

HAL is a multi-disciplinary open access archive for the deposit and dissemination of scientific research documents, whether they are published or not. The documents may come from teaching and research institutions in France or abroad, or from public or private research centers.
L'archive ouverte pluridisciplinaire HAL, est destinée au dépôt et à la diffusion de documents scientifiques de niveau recherche, publiés ou non, émanant des établissements d'enseignement et de recherche français ou étrangers, des laboratoires publics ou privés. 


\title{
Charged particle dynamics in a tangential discontinuity
}

\author{
Roch Smets ${ }^{1}$ \\ NASA Goddard Space Flight Center, Greenbelt, Maryland
}

\begin{abstract}
We investigate the regularity of particle motion in a magnetopause-like magnetic field topology for a southward interplanetary magnetic field case. For that issue, a rotation of $180^{\circ}$ of the magnetic field direction through the magnetopause is considered, while its total magnitude remains constant. Our main interest is to emphasize the different dynamical regimes for charged particles when their Larmor radii are of the order of the thickness of the magnetic field reversal layer. When the interface is a tangential discontinuity, it is shown that particle dynamics is regular, whereas the introduction of a small component of the magnetic field, normal to the interface, results in weakly stochastic dynamics. A new parameter $\kappa_{R L}$ is introduced to organize the charged particle dynamics. When $\kappa_{R L}$ is greater than 1 , particle motion is adiabatic, and when $\kappa_{R L}$ is smaller than 1 , particle motion is nonadiabatic, its magnetic moment being possibly either damped or unchanged when interacting with the discontinuity. Implications for the pitch angle distribution of a population interacting with such a tangential discontinuity will be discussed.
\end{abstract}

\section{Introduction}

A large number of studies have previously been dedicated to nonadiabatic motion of charged particles in the magnetotail. The thin current sheet observed in the nightside of the magnetosphere is on the origin of a tightly curved magnetic field. For the thermal ion population of the plasma sheet $(1 \mathrm{keV})$ the curvature radius $R_{C}$ of the magnetic field lines at the equator is of the order of the Larmor radius $\rho_{L}$ in the near tail (at $\sim 8$ $R_{E}$ ) and is several times smaller in the far tail (after 50 $R_{E}$ ). Considering a magnetic field along the Sun-Earth direction reversing on a small scale length compared to the Larmor radius, the so-called Harris sheet (HS) [see Harris, 1962], Speiser [1965] showed that charged particles remain infinitely trapped in the neutral sheet, exhibiting a fast oscillation in the direction perpendicular to the HS. The addition of a small component of the magnetic field in the south-north direction (normal to the HS that we will call the modified HS) lead to untrap these particles. In such motion the magnetic moment is not conserved, but the action integral $I_{z}=(2 \pi)^{-1} \oint \dot{z} d z$ (where $z$ is the direction of oscillation) is an adiabatic invariant [see Speiser, 1970] and Sonnerup [1971] gave its analytic form in a pure HS. This motion is called quasi-adiabatic or super-adiabatic motion.

\footnotetext{
${ }^{1}$ Permanently at Centre d'étude des Environnements Terrestre et Planétaires, Centre National de la Recherche Scientifique, Vélizy, France.
}

Copyright 2000 by the American Geophysical Union.

Paper number 1999JA000173.

0148-0227/00/1999JA000173\$09.00
Performing Poincaré surfaces of section (SOS) displaying the particles' position in the phase space when crossing the plane of minimum magnetic field, Chen and Palmadesso [1986] found different behaviors depending on the particles' Hamiltonian. When $R_{C} \sim \rho_{L}$, the Poincaré SOS are uniformly filled, which is characteristic of a chaotic dynamics. However, when $R_{C}<\rho_{L}$, there are some uniformally filled regions and some regions where the crossing points draw closed curves. These closed curves (also called Kolmogorov Arnold Moser (KAM) surfaces) prove the existence of islands of stability, related to the existence of an adiabatic invariant. Karimabadi et al. [1990] displayed Poincaré SOS associated to different magnetotail configurations. Burkhart et al. [1995] investigated the influence of the $z$ component of the magnetic field in the Poincaré SOS using a modified HS and Büchner and Zelenyi [1991] and Holland et al. [1996] studied the influence of a small $y$ component of the magnetic field in the same topology.

Aiming to classify the possible orbits as to their integrability, Büchner and Zelenyi [1989] proposed the curvature parameter $\kappa$ (that we will call later $\kappa_{B Z}$ ), defined as

$$
\kappa_{B Z}=\left[\frac{R_{C \min }}{\rho_{L \max }}\right]^{1 / 2},
$$

where $R_{C \min }$ is the minium curvature radius of the magnetic field lines and $\rho_{L \max }$ is the maximum Larmor radius. For $\kappa_{B Z}>3$ the motion is essentially adiabatic, for $\kappa_{B Z} \sim 1$ the motion is chaotic, and for $\kappa_{B Z} \ll 1$ the motion is either chaotic or super-adiabatic. Chaotic system has to be understood in the sense of high sensitivity to initial conditions. Strictly speaking, this system is a chaotic scattering system [see, e.g., Eckhardt, 
1988]. The magnetic field being constant, particles are not constraint to stay in the stochastic region (in the discontinuity) and escape in the asymptotic region (out of the discontinuity) where the magnetic field is uniform and the particle motion adiabatic. Chen [1992] put forward the divergence of two initially nearby orbits in a modified HS computing the average exponent divergence rate (AEDR), which is a modification of the Lyapunov procedure.

Berchem and Russell [1982a] showed on the basis of ISEE 1 and 2 observations that the magnetopause thickness is $\sim 800 \mathrm{~km}$, which is of the order of the $1 \mathrm{keV} \mathrm{H}^{+}$ Larmor radius (see also Le and Russell [1994]). We thus expect finite Larmor radius effects at the magnetopause. Examining Explorer 12 magnetic field measurments, Sonnerup and Cahill [1967] showed that in most of the magnetopause crossing, the magnetic field component, normal to the magnetopause, is very weak, and the magnetopause is thus essentially a tangential discontinuity (TD), the magnetic field being always tangential to the magnetopause. Studying the magnetopause polarization, Berchem and Russell [1982b] also identified it as a TD and showed a rotation of the magnetic field from its asymptotic values in both sides of the magnetopause. Papamastorakis et al. [1984] obtained the same result for large field angle rotation and concluded that even for strong southward interplanetary magnetic field (IMF), when reconnection is suspected, the discontinuity is tangential and not rotational.

Several studies have been dedicated to particle dynamics in magnetopause-like magnetic field geometry. Some of the magnetic topology used in testparticle calculations where similar to the one used in the tail during reconnection events with gradient and possible annihilation of the magnetic field [see, e.g., Speiser et al., 1981; Curran et al., 1987; Curran and Goertz, 1989]. Swift and Lee [1983] studied numerically the magnetopause polarization as its stability for different initial conditions of rotational discontinuity. However very little attention has been devoted to tangential discontinuities and to the associated charged particle dynamics that seems the most relevant magnetic topology for the quiet magnetopause. Using the Hamiltonian mechanics, the stochasticy of charged particles in a slab geometry with a laminar magnetic field, such as the one observed at the magnetopause, is investigated.

The equations of motion in the frame of Hamiltonian mechanics will be introduced in section 2 , and the implications for charged particle dynamics will be discussed. Both TD and modified TD (TD with a small component of the magnetic field normal to the interface) will be investigated. The new parameter $\kappa_{R L}$ will be introduced in section 3 to classify the different behaviors, and phase space structure will be discussed using computed Poincaré SOS. Section 4 will be a focus on the origin of stochasticity of charged particle motions in a modified TD. Section 5 will present a comparison between dynamics in a modified HS and in a modified TD. Section
6 will summarize the obtained results and the implications for the pitch angle distributions at the traversal of the discontinuity will be introduced. This study is focused on the high magnetic shear case with a field rotation angle of $180^{\circ}$. Other cases in which the magnetic shear is smaller than $180^{\circ}$ will be also discussed in section 6 .

\section{Particle Dynamics}

The simplest model to describe the magnetic field at the magnetopause (assuming a rotation of $180^{\circ}$ from a southward magnetic field in the magnetosheath to a northward magnetic field in the magnetosphere) when there is no gradient of the magnetic field magnitude across this interface is

$$
\begin{aligned}
& b_{x}=b_{0} \cos (\pi y / 2 L) \\
& b_{y}=0 \\
& b_{z}=b_{0} \sin (\pi y / 2 L)
\end{aligned}
$$

with $b_{0}$ being the asymptotic value of the magnetic field and $L$ being the half thickness of the magnetopause. Equations of system $(2 \mathrm{a}-2 \mathrm{c})$ are valid for $-L<y<$ $+L$. For $y<-L$ (respectively $y>+L$ ), $b_{z}=-b_{0}$ (respectively $b_{z}=+b_{0}$ ), with $b_{x}=b_{y}=0$. The vector potential associated to this magnetic field vector is

$$
\vec{A}=\frac{2 L b_{0}}{\pi}[\cos (\pi y / 2 L) \hat{x}+\sin (\pi y / 2 L) \hat{z}]
$$

with the Coulomb gauge. Calculations being less tedious using dimensionless coordinates, distances are normalized to the half thickness of the magnetopause $L$ and time to the gyrofrequency $\Omega=q b_{0} / m$ of the particles of mass $m$ and charge $q$. In the absence of an electric field the Lagrangian is thus given by

$$
L=\frac{1}{2}\left(\dot{x}^{2}+\dot{y}^{2}+\dot{z}^{2}\right)+\frac{2}{\pi}[\dot{x} \cos (\pi y / 2)+\dot{z} \sin (\pi y / 2)] .
$$

The three canonical momenta are thus

$$
\begin{aligned}
& P_{x}=\dot{x}+A_{x} \\
& P_{y}=\dot{y} \\
& P_{z}=\dot{z}+A_{z}
\end{aligned}
$$

with $A_{x}=(2 / \pi) \cos (\pi y / 2)$ and $A_{z}=(2 / \pi) \sin (\pi y / 2)$. The Lagrangian does not depend on $x$ and $z$, and as a result, $P_{x}$ and $P_{z}$ are constants of the motion $\left(d_{t} P_{x}=0\right.$ and $\left.d_{t} P_{z}=0\right)$. Using the Legendre transformation $H=$ $\mathbf{P r}-L$ (where $\mathbf{r}$ denotes the generalized coordinates), the Hamiltonian is

$$
H=\frac{1}{2}\left[\left(P_{x}-A_{x}\right)^{2}+P_{y}^{2}+\left(P_{z}-A_{z}\right)^{2}\right]
$$

that is also a constant of the motion $\left(d_{t} H=0\right)$ as it does not explicitly depend on time. A system with 3 degrees of freedom is known to be integrable if there are three 
constants of the motion that are in involution, meaning that their Poisson brackets vanish [see, e.g., Lichtenberg and Liebermann, 1983]. It is easily verifiable that it is here the case. Hence there exists a frame in which $H, P_{x}$, and $P_{z}$ are the new canonical momenta, the solution of the Hamilton's equations being trivial in this frame. Particle dynamics in a pure TD is thus regular and predictible.

The system described in this study can be compared to the one studied by Speiser [1965] and Sonnerup [1971] in a HS. In the absence of a small $z$ component of the magnetic field normal to the neutral sheet, particle motion can be either adiabatic or super-adiabatic, whatever the value of $\kappa_{B Z}$ (the higher $\kappa_{B Z}$, the thinner the super-adiabatic layer). The corresponding orbits are called noncrossing orbits and meandering orbits, respectively (see, e.g., Figure 2 of Sonnerup [1971]). However, in a pure HS, there is no phase space regions where one can find both adiabatic and super-adiabatic dynamics (involving transition regions) for the same orbit. In the same way, for a pure TD (no component of the magnetic field normal to the interface), particles initially far from the interface will never get close to it ( $\mu=m V_{\perp} / q b_{0}$ being conserved) and particles initially close to the interface will never escape from it. However, annihilation of the magnetic field in the HS results in the existence of a super-adiabatic layer, whatever the value of the Hamiltonian, whereas this layer can vanish in the TD because of the absence of gradient in the total magnetic field magnitude. In the case where particles are close to the interface (in a TD), they can experience both meandering orbits (a constant of the motion $I_{y}=(2 \pi)^{-1} \oint \dot{y} d y$ can be defined in the same way as in a HS as shown in section 4) or adiabatic orbits depending on their Hamiltonian value.

With the magnetic topology defined by (2a-2c), particle dynamics is regular and the associated Hamiltonian system is integrable. This conclusion is valid under the assumption that the electric field (not taken into account in this study) is also not able to drive particles close to the discontinuity. The convection and corotation electric field usually drive particles tangentially to the magnetopause, unless there exists some reconnection region. Thus the electric field can hardly be responsible for an evolution from noncrossing to meandering orbit.

In the magnetopause data a component of the magnetic field, even small, is generally observed in the direction normal to the interface. In a similar way to the tail configuration, this small component of the magnetic field can carry particles initially far from the discontinuity in its vicinity. Thus transient orbits in which particles experience a transition from noncrossing orbits to meandering orbits are expected. The equation (2b) is modified with $b_{y}=b_{n}$, where $\delta=b_{n} / b_{0}$ is small, to study the effect of such a small component of the magnetic field on particles dynamics. This topology is referred to as the modified TD.

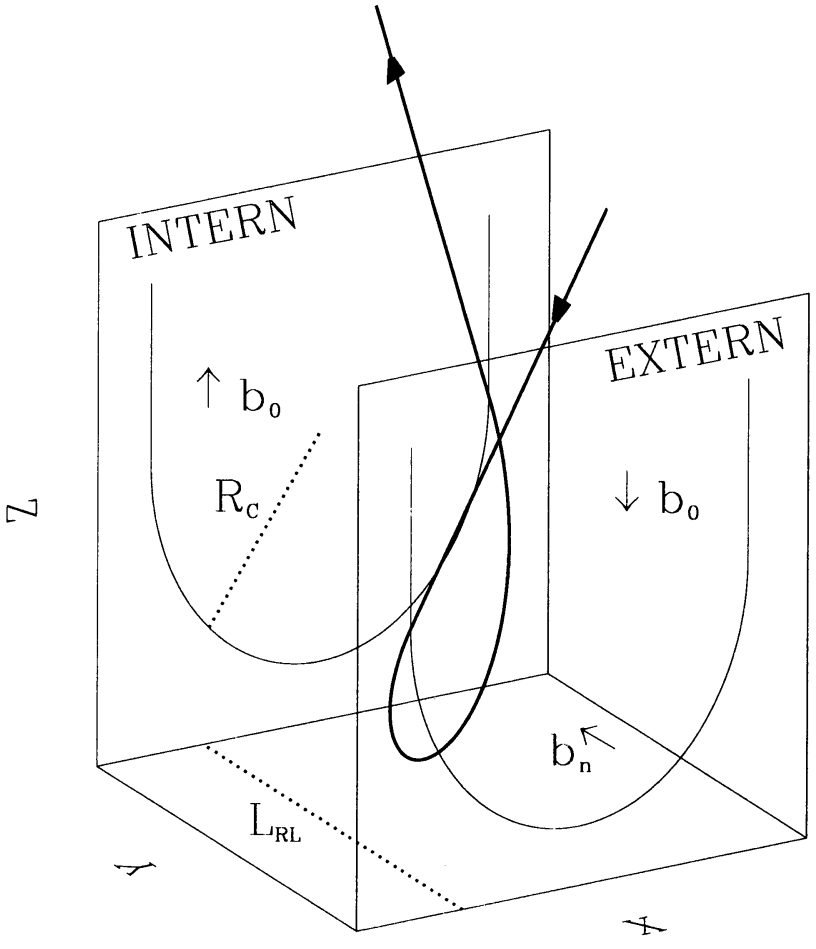

Figure 1. Solid thick line presents a magnetic field line in three dimensions. The arrow indicates the southward orientation of the magnetic field in the magnetosheath and its northward orientation in the magnetosphere. Dashed lines present the local curvature radius $R_{C}$ and the characteristic scale length $L_{S}$.

It has to be noted that a rotational discontinuity has by definition a component of the magnetic field normal to the discontinuity of same magnitude in each side of the discontinuity (satisfying the Rankine-Hugoniot jump conditions). There is no assumption on its magnitude compared to the total magnetic field magnitude. To make a point that we consider in this study a normal component of the magnetic field of first order compared to the tangential magnetic field, we call this topology a modified TD as it is close to a pure TD. The resulting shape of the magnetic field lines is depicted in Figure 1. The solid thick line is a magnetic field line oriented southward for $y<-L$, and northward for $y>+L$. The two vertical planes are defined by $y=-L$ and $y=+L$, in which the solid lines are the projection of the magnetic field lines. This depicts the duskside of the magnetopause.

In this system the new vector potential then depends on the choice of the gauge. With the Coulomb gauge,

$$
\begin{aligned}
& A_{x}=2 / \pi \cos [\pi y / 2]+\delta z \\
& A_{z}=2 / \pi \sin [\pi y / 2]
\end{aligned}
$$

The $z$ coordinate is no longer cyclic, as the Lagrangian depends on $z$, and $P_{z}$ is no longer a constant of the motion. However, knowing only two constants of 

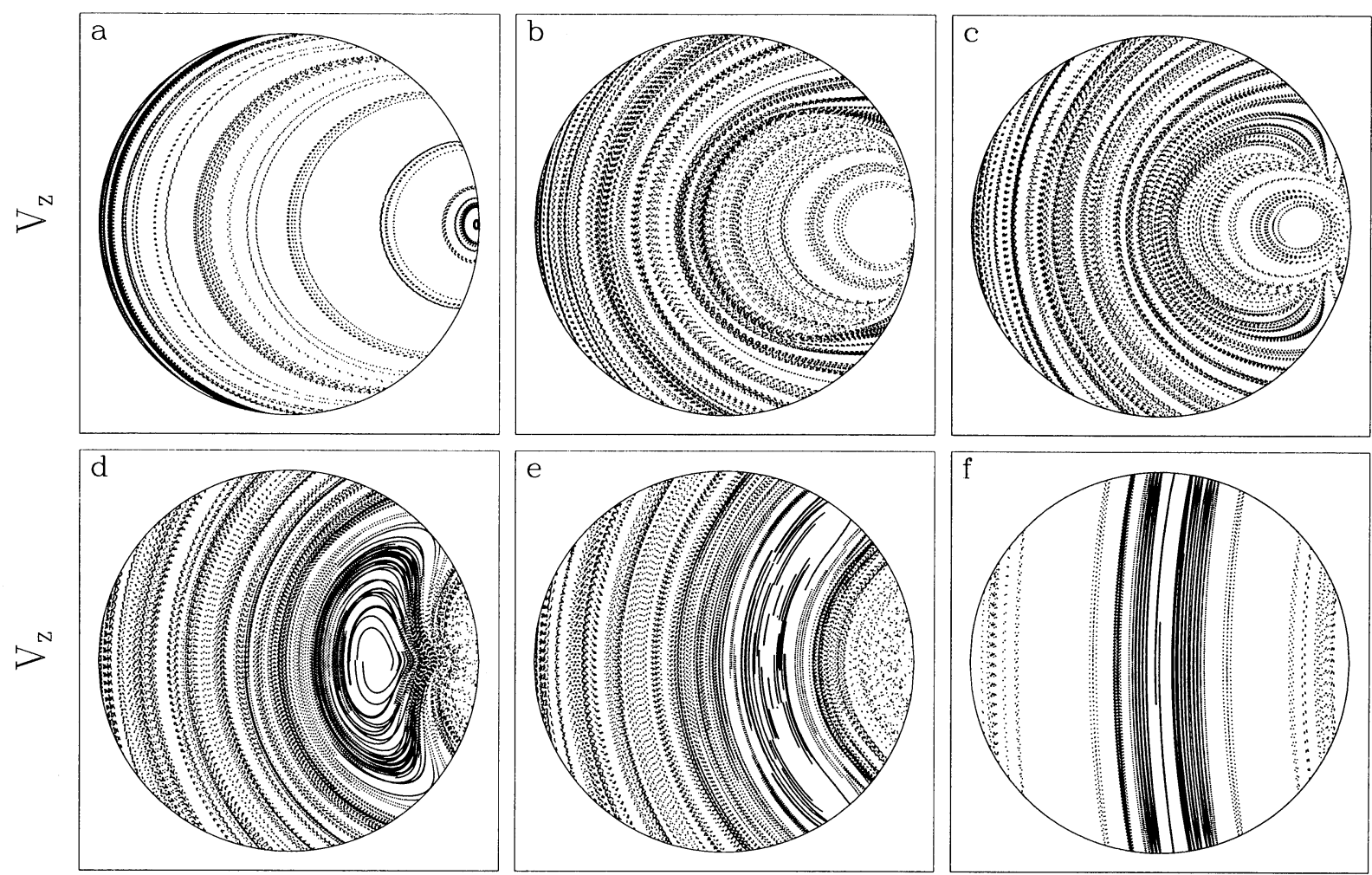

Z

Z

Z

Figure 2. Poincaré surface of section in the $(z, \dot{z})$ plan: (a-f) obtained at $\kappa_{R L}=0.3,0.8,1.0$, $1.2,1.4$, and 3.0 , respectively.

the motion using elementary methods does not rule out the existence of a third (that can be in involution with the two others). The easier way to predict the integrability of the system is to compute Poincaré surfaces of section [see, e.g., Lichtenberg and Liebermann, 1983].

\section{The $\kappa_{R L}$ Parameter and the Poincaré Surfaces of Section Structures}

The Poincaré SOS display the phase space position of a set of particles when crossing a given plane. The set of particles is chosen to cover the whole phase space, assuming that they all have the same values for the constants of the motion ( $H$ and $P_{x}$ for a modified 'TD). The plane is generally chosen for its symmetric properties and the Poincare SOS presented in this study are built in the $y=0$ plane.

As different types of behavior are expected depending on the value of the normalized Hamiltonian, the new dimensionless parameter $\kappa_{R L}$ is introduced

$$
\kappa_{R L}=\left[\frac{L_{R L}}{\rho_{L \text { ma.x }}}\right]^{1 / 2}
$$

where $L_{R L}$ is the thickness of the reversal layer and $\rho_{\text {Lmax }}=m V / q b_{0}$ is the maximum Larmor radius. In the present case, $L_{R L}=L$, the half thickness of the magnetopause. As $\kappa_{B Z}$ allows a classification of the different orbits depending on the importance of the curvature of the magnetic field lines, the $\kappa_{R L}$ parameter is proposed to organize the possible orbits in topology with just a change of the magnetic field direction and no gradient of its total magnitude. In the modified TD case, $\kappa_{R L}=\left[L q b_{0} / m V\right]^{1 / 2}$. Section 5 will be dedicated to the comparison between $\kappa_{B Z}$ and $\kappa_{R L}$.

Figure 2 displays Poincaré SOS in the $(z, \dot{z})$ plan obtained for different values of $\kappa_{R L}$. These Poincaré SOS have been built with $P_{x}=0$ and $\delta=0.01$. The first Poincaré SOS in Figure 2a displays the phase space structure at $\kappa_{R L}=0.3$. The computed crossing points draw curves (being the two-dimensional reduction of KAM surfaces). However, it can be seen that except at the very right part of the Poincaré SOS, those curves are not closed. The Poincaré SOS is just a collection of these arched curves. In order to understand these features a focus on the associated orbits is needed.

Figure 3 displays three particle orbits in a modified TD. The shaded region represents the $y=0$ plan, and the solid and dotted thick lines represent the particle position for $y<0$ and $y>0$, respectively. Hence the particle position at $y=0$ (on the shaded plane) are the one displayed in the Poincaré SOS. The orbit presented in Figure $3 \mathrm{a}$ is obtained at $\kappa_{R L}=0.49$, which corresponds to $H=10$, other parameters being set to 

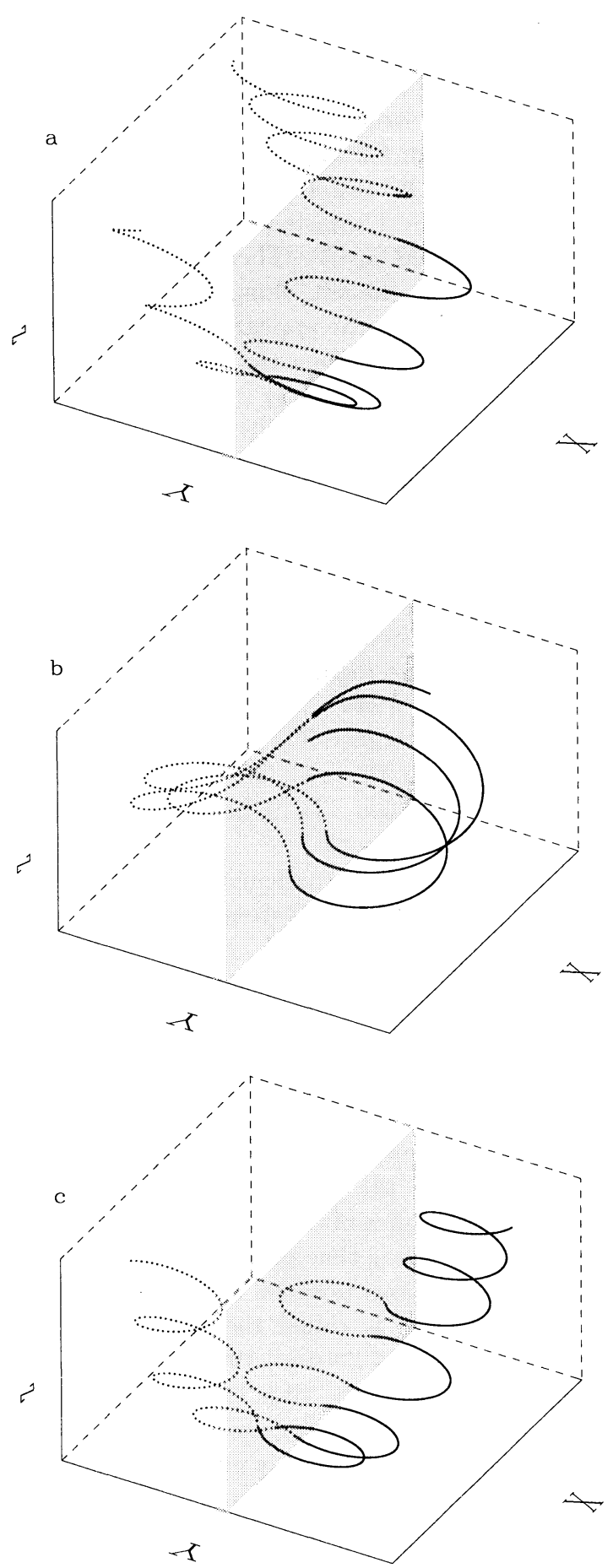

Figure 3. Particle orbits in a modified tangential discontinuity. The shaded region indicated the $y=0$ plan, and the solid and dotted thick line indicate the particle position in the $y<0$ and $y>0$ region, respectively. (a) Nonescaping particle, (b) trapped particle, and (c) escaping particle.

$P_{x}=0$ and $\delta=0.1$ (the two other orbits of Figure 3 are obtained with the same values of $\kappa_{R L}, P_{x}$ and $\delta$ ). There is two adiabatic sequences for which the particle has a regular cyclotron motion around the magnetic field lines and a super-adiabatic motion for which the particle is meandering around the $y=0$ plan. This type of orbit is somewhat similar to the Speiser orbits [see Speiser, 1965] in a modified HS. As the parameter $\delta$ is small, there is a clear decoupling between the fast oscillation in the $y$ direction, owing to the reversal of the $z$ component of the magnetic field in each sides of the $y=0$ plane, and the slow gyration around the $b_{y}$ component in the $(x, z)$ plane.

This is on the origin of the arched curves in the Poincaré SOS. When getting close to the TD, $z$ is decreasing as the magnitude of $\dot{z}$. Reaching the minimum value of $z, \dot{z}$ gets null, change its sign, and then its magnitude enhanced with the opposit sign while $z$ is still increasing. A point that has to be noted here is that at $\kappa_{R L}=0.3$, almost all these particles have this transient motion near the discontinuity. It is not the case for the marginal amount of particles that depict the small closed curves in the very right part of the Poincaré SOS of Figure 2.

Figure $2 b$ depicts the Poincaré SOS computed for $\kappa_{R L}=0.8$. Compared to the previous Poincaré SOS, the arched curves get fuzzy in the way that the crossing points are more dispersed than in Figure 2a and do not draw perfect lines. Furthermore, the closed features (at the right part of the plot) are developing in the phase space. As already mentioned at the begining of this section, KAM surfaces lies on the existence of 3 constants of the motion, for a system with 3 degrees of freedom. $H$ and $P_{x}$ being conserved, the third one is the action integral $I_{y}=(2 \pi)^{-1} \oint \dot{y} d y$. We will show numerically in next section that $I_{y}$ is conserved only when $\kappa_{R L} \ll 1$. The closed structures in Poincaré SOS were close to KAM surfaces for $\kappa_{R L}=0.3$ as $I_{y}$ was conserved but get fuzzy at $\kappa_{R L}=0.8$ as $I_{y}$ is not conserved anymore.

The origin of these closed structures lies on the existence of trapped orbits in the modified TD. Associated to the meandering motion in the $y$ direction, the particle experience a slower gyromotion around the small $y$ component of the magnetic field. Instead of experiencing half a turn around this small $y$ component as in Figure 3a, trapped particles keep on gyrating around this component through the time. An example of this type of orbit is presented in Figure $3 \mathrm{~b}$. Even if just a fraction of the orbit is depicted for clearness, the particle stays trapped in the modified TD. The corresponding features in the Poincaré SOS are not closed curves as $I_{y}$ is still not conserved but rather look like seashell. This spiral structure will become clearest in Figure 2c. The amount of trapped particles in the $T D$ is increasing with $\kappa_{R L}$.

Figure $2 \mathrm{c}$ is the Poincaré SOS computed for $\kappa_{R L}=$ 1.0. The two types of structures mentioned above are still present: the arched curves at the left part of the Poincarré SOS are still fuzzy, and the seashell-like structures are developing in phase space. The explanation for theses structures is the same as discussed above. But it has to be noted that the seashell-like structure 


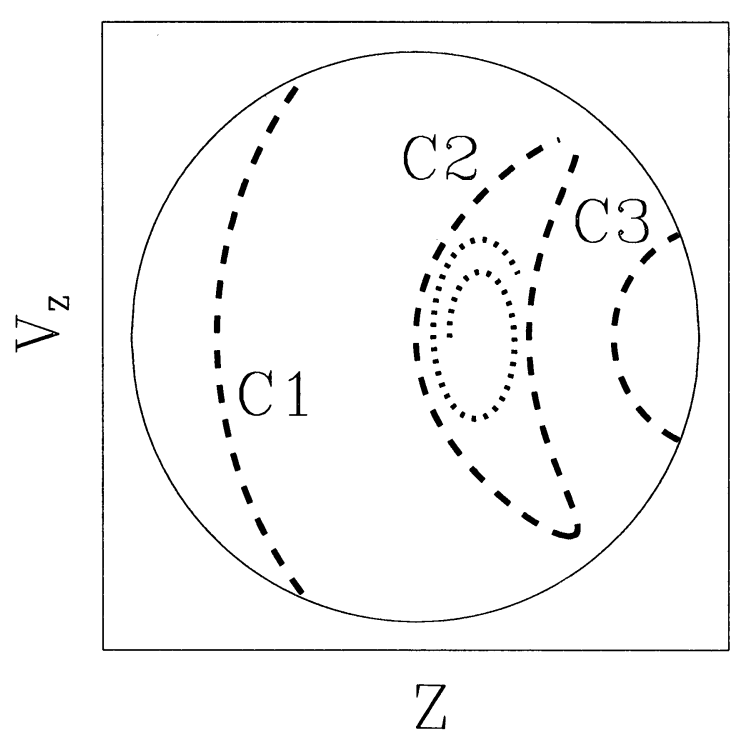

Figure 4. Schematic structure of a Poincaré surface of section in the $(z, \dot{z})$ plan. $\mathrm{C} 1$ and $\mathrm{C} 3$ regions (in dashed line) are associated to untrapped orbits, while C2 is associated to trapped orbits. The dotted line represent the seashell-like structure associated to weakly nonregular orbits.

is shifting to the left. Another point is that even if the arched curves are fuzzy, they still are arch shaped. That why we can not talk about chaos but rather on weak stochasticity.

Figure 2d is the Poincare SOS computed for $\kappa_{R L}=$ 1.2. As the $\kappa_{R L}$ value is increasing, it is clear that the seashell-like structure is occupying more space in the whole phasespace. These structures stay localized at the large values of $z$ and are now compressed on their right side. The very right part of the Poincaré SOS is then composed of new arched curves that are developing and shifting to small $z$ values. These arched curves are very fuzzy but will become clearest at higher $\kappa_{R L}$ value. This can be seen in Figure 2e, displaying the Poincaré SOS computed for $\kappa_{R L}=1.4$. As the seashelllike structures are compressed, they get elongated in the $\dot{z}$ direction. These structures are thus not closed anymore and become arched curves as the tip of the seashell-like structures gets out of the Poincaré SOS. As $\kappa_{R L}$ is increasing, seashell-like structures are opening. This means that increasing $\kappa_{R L}$ results in detrapping of particles in the modified TD.

Figure $2 \mathrm{f}$ is the Poincaré SOS computed for $\kappa_{R L}=$ 3.0. The seashell-like structure has been so compressed that they all became arched curves. This is the adiabatic regime for which particles' magnetic moment is conserved when interacting with the modified TD. The magnetic moment is now the third constant of motion, dynamic is regular, and the Poincaré SOS is just composed of KAM surfaces. It has to be said that the blank in the Poincaré SOS is due to the sampling of particles in order to make the plot clearest but could be filled with a different sampling.
The features discussed above are summarized in Figure 4. This is a schematic view of the Poincaré SOS. At low $\kappa_{R L}$ value the Poincare SOS is just made of arched curves as in structure $\mathrm{C} 1$. When increasing $\kappa_{R L}$, closed curves like in $\mathrm{C} 2$ appear at the very right part of the Poincare SOS and shift to the left as $\kappa_{R L}$ increases. At $\kappa_{R L} \sim 1$, seashell-like structures appear like the one enclosed in C2. Then as $\kappa_{R L}$ continue to increase, $\mathrm{C} 2$ shift to the left, elongate in the $\dot{z}$ direction and get compressed by the appearing C3 structure that are also arched curves. Then the tips of $\mathrm{C} 2$ get out of the Poincaré SOS, and for $\kappa_{R L}>3$, Poincaré SOS is just made of $\mathrm{C} 1$ and $\mathrm{C} 3$ structures.

There are several conclusions arising from the study of these Poincaré SOS:

1 . The $\kappa_{R L}$ is a new parameter that can organize particle dynamics in modified TD. When $\kappa_{R L} \gg 1$, particle motion is regular, the adiabatic moment being the third constant of motion (with $H$ and $P_{x}$ ). When $\kappa_{R L} \ll 1$, particle motion is also regular, $I_{y}=(2 \pi)^{-1} \oint \dot{y} d y$ being the new constant of motion associated to the meandering motion in the modified TD.

2. Depending on the $\kappa_{R L}$ value, particles can be trapped in the modified TD: it happens for $\kappa_{R L} \sim 1$. This system is then chaotic.

3 . The other particles experience just one interaction with the modified TD. This system is a chaotic scattering system.

The consequences of these conclusions will be discussed in section 6 .

\section{Separatrix Crossing}

When particle dynamics is regular (either adiabatic or super-adiabatic), the third constant of the motion guarantees the existence of KAM surfaces in the Poincaré SOS (particle motion is thus predictible). Stochasticity arises at the transitions between different types of motion. This sequence is called the separatrix crossing [see, e.g., Büchner and Zelenyi, 1990].

Figure 5 (bottom) is the time evolution of the $y$ position of a particle with $\kappa_{R L}=0.45, P_{x}=0$ and $\delta=0.2$. Figure $I_{y}=(2 \pi)^{-1} \oint \dot{y} d y$ and (dotted line) $\mu=m V_{\perp} / q b$, both in normalized units. The shaded regions are displayed to allow a comparison with Figure 6 and separate regions 1,2 , and 3 . It is clear that in the adiabatic sequence (region 1 ), $\mu=m V_{\perp} / q b$ is the third constant of the motion, whereas in the superadiabatic sequence (region 3 ), $I_{y}=(2 \pi)^{-1} \oint \dot{y} d y$ is the new one. The existence of this third constant of the motion vanishes during the switch between the two sequences (region 2). To emphasize the importance of region 2 and point out that it is on the origin of stochasticity in particle dynamics, a brief and qualitative analysis of the Hamiltonian equations is required.

Using (5a-5c) and (6), the first set of Hamiltonian's equations is

$$
\ddot{x}=(\pi / 2) \dot{y} A_{z},
$$




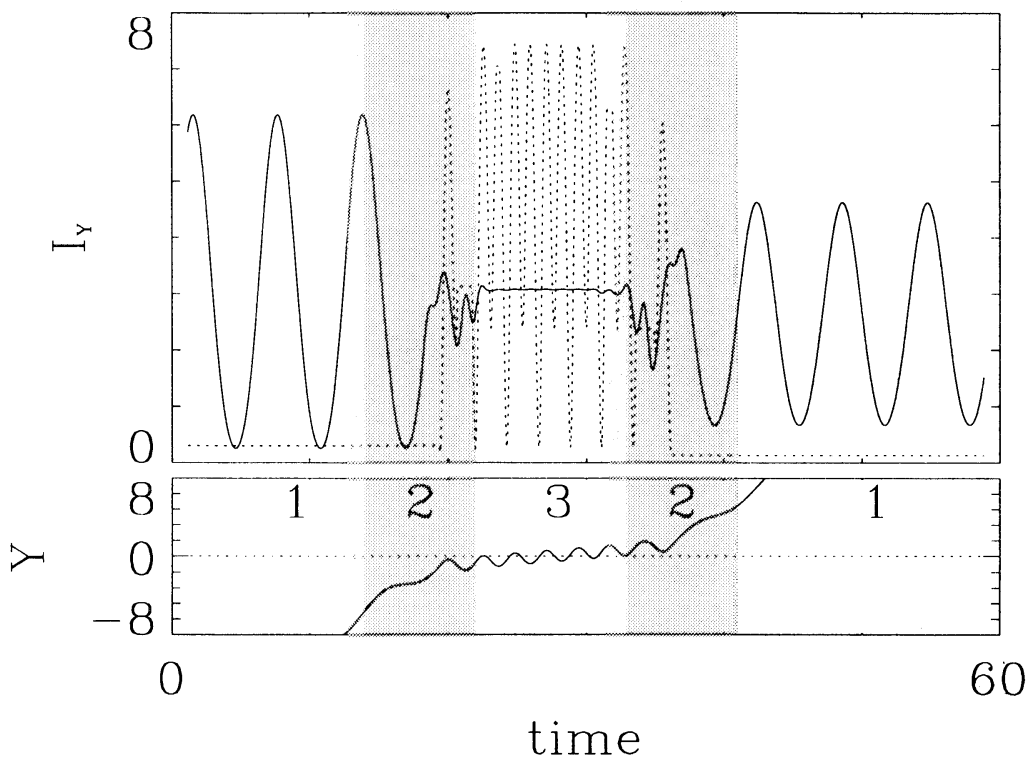

Figure 5. (top) (solid line) Action integral value and (dotted line) the magnetic moment evolution of the particle depending on time; (bottom) time evolution of the $y$ position.

$$
\begin{aligned}
\ddot{y} & =(\pi / 2) \dot{z} A_{x}-(\pi / 2) \dot{x} A_{z} \\
\ddot{z} & =(-\pi / 2) \dot{y} A_{x} .
\end{aligned}
$$

During the meandering sequence near the $y=0$ plane the motion can be seen as the superposition of a fast oscillation in the $y$ direction and a slow gyromotion in the $(x, z)$ plane around the small $b_{y}$ component. On the timescale of the fast oscillation, displacement due to the slow gyromotion in the $(x, z)$ plan is of first order and thus has $z \sim \mathrm{C}^{\text {st }}$ near $y=0$. Using a Taylor series expansion near $y=0$, (9b) is of the form

$$
\ddot{y}=K \dot{x} y
$$

( $K$ being a constant). Defining $\tau_{\dot{x}}$ and $\tau_{y}$ as the timescale of variation of $\dot{x}$ and $y$, respectively, (10) will be nonlinear when $\tau_{\dot{x}} \sim \tau_{y}$, linear in $\dot{x}$. when $\tau_{\dot{x}} \ll \tau_{y}$ and linear in $y$ when $\tau_{\dot{x}} \gg \tau_{y}$.

We computed at each time, $t_{i}$, the period of time, $\Delta t$, neecied to get $\Delta \dot{x}_{i} / \dot{x}_{i}>\epsilon$ or $\Delta y_{i} / y_{i}>\epsilon$ (with $\Delta u_{i}=u\left(t_{i}+\Delta t\right)-u\left(t_{i}\right)$ and $\left.\epsilon=0.5\right), \Delta t$ representing $\tau_{\dot{x}}$ and $\tau_{y}$, respectively. We have thus defined the "Lorentz number" as

$$
N_{L}=\frac{1}{\epsilon}\left[\frac{\Delta \dot{x}}{\dot{x}}+\frac{\Delta y}{y}\right],
$$

which can be seen as the "order of the polynomial" in the right-hand side of (10). When $N_{L}$ is $\sim 1,(10)$ is a polynom of first order in $\dot{x}$ or in $y$ (depending on the scaling between $\tau_{\dot{x}}$ and $\tau_{y}$ ). When $\tau_{\dot{x}} \sim \tau_{y}$, (10) is nonlinear and can be seen as a second order polynom of a variable depending on $\dot{x}$ and $y$. We suspect emergence of stochasticity in regions where $N_{L}$ reaches 2 , whereas when $N_{L}$ is close to 1 , dynamics should be regular. Figure 6 depicts the evolution of this number (top) and the evolution of the $y$ coordinate (bottom) over time for $\kappa_{R L}=0.45$. This orbit is the same as the one presented in Figure 5.

Three different regions appear in Figure 6:

Region 1, where the particle motion is adiabatic, $\mu$ is conserved, $N_{L}$ is close to 1 and the system is quite linear; Region 2 (indicated by the light gray rectangle) where the particles get close to the interface. In this transition region, between adiabatic motion and superadiabatic motion, $N_{L}$ reaches 2 and the system is nonlinear; Region 3 , near the interface where the particle motion is super-adiabatic. $I_{y}$ is conserved, $N_{L}$ is close to 1 and the system is quite linear.

Region 2 in Figure 6 is a visual representation of the separatrix between adiabaticity and super-adiabaticity. When flowing through region 2 , evolution of the phase space coordinates is stochastic.

The situation in the modified TD is quite different from the one in the modified HS. As in the modified TD the magnetic field magnitude is uniform, each particle experience just two crossings of the separatrix, each of them being associated $t o$ a chaotic scattering. In the modified HS the magnetic field gradient and the resulting bounce motion of particles leads to a succession of chaotic scattering. Furthermore, not only the number of chaotic scattering plays a role, but the nature of this scattering is different. This will be discussed in section 5 . In this case, even if $I_{z}$ is conserved during the meandering sequences, its value is not the same in each of the meandering sequences, because of its stochastic determination when crossing the separatrix. Hence the crossing points of the Poincaré SOS in the modified HS cannot draw any KAM curves. This is almost the case for the resonant energies (defined as the energies for which the magnetic moment is conserved after interacting with the modified HS) and the resulting Poincaré surfaces of 


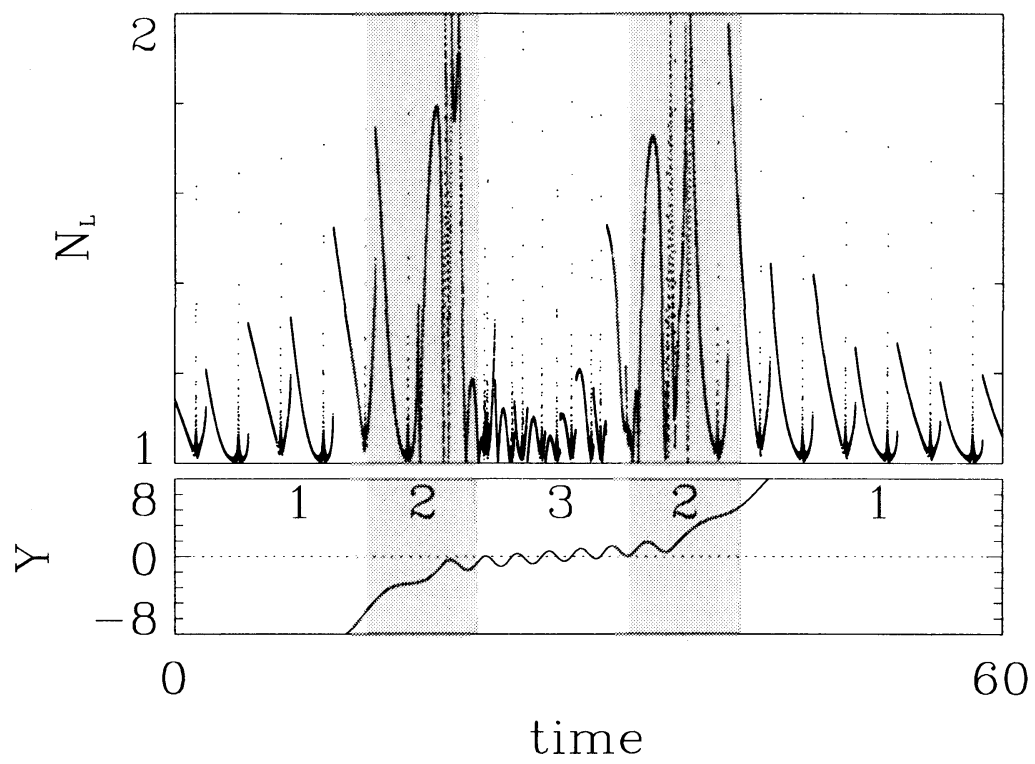

Figure 6. (top) $N_{L}$ value (defined by (11)) and (bottom) $y$ coordinate of the particle depending on time. In region 1 and 3 the system is weakly nonlinear $\left(N_{L}\right.$ is close to 1$)$, and in region 2 indicated by light gray, the system is strongly nonlinear $\left(N_{L}\right.$ is close to 2).

section are more structured with the emergence of tentacles [see, e.g., Chen and Palmadesso, 1986].

Because there is only two separatrix crossings in the modified TD, the evolution of the particle position in phase space is weakly stochastic. At the first crossing the initial value of the action integral $I_{y}$ is determined stochastically. As this value will not be the same for each particle, KAM curves cannot be obtained. However, the set of curves obtained from all the particles are close to one another, meaning that the different values of the action integrals are close. The phase space is well-organized, and the Poincaré SOS are not densely filled. Chaos cannot develop in modified TD, because of the limited number of interactions of the particles with the magnetic boundary.

\section{Relation Between $\kappa_{B Z}$ and $\kappa_{R L}$}

There is a duality in the relative importance of magnetic field line curvature and finite size of the reversal layer effects between the modified HS and the modified TD. In the modified HS, $\vec{b}=b_{0}(z / L) \hat{x}+b_{n} \hat{z}$. The reversal layer thickness is $L_{R L}=L$ and the curvature radius of the magnetic field lines is $R_{C}=L \delta$, with $\delta=b_{n} / b_{0}$. Value $\delta$ being of the order of 0.1 or less in the tail, the curvature effects of the magnetic field lines will be dominant. In this topology, $\kappa_{B Z}=\delta \kappa_{R L}$ and particle dynamics will be essentially governed by the $\kappa_{B Z}$ parameter. In the modified TD, $\vec{b}=b_{0} \cos [\pi y / 2] \hat{x}+b_{n} \hat{y}+b_{0} \sin [\pi y / 2] \hat{z}$. In this case, $L_{R L}=L$, and $R_{C}=L / \delta$ ( $\delta$ being defined as in the former case). In Figure 1 the curvature radius and the reversal layer are indicated by thick dot- ted lines and labeled $R_{C}$ and $L_{R L}$, respectively. Hence $\kappa_{B Z}=\delta^{-1 / 2} \kappa_{R L}$, and effects associated to the finite size of the reversal layer become dominant as $\delta \ll 1$. Thus we could expect a differentiation of the possible orbits depending on the $\kappa_{R L}$ parameter value.

Several studies have been devoted to charged particle motion in a reversal magnetic field topology (like a HS or modified HS) in the presence of a shear field [see, e.g., Karimabadi et al., 1990; Büchner and Zelenyi, 1991; Zhu and Parks, 1993; Martin et al., 1994; Holland et al., 1996]. Focusing on the magnetotail, this corresponds to a component of the magnetic field in the $y$ direction of the order of the $z$ component in quiet time, which become larger or dominant in flux ropes. Büchner and Zelenyi [1991] proposed the two parameter $\kappa_{n}$ and $\kappa_{s}$ associated to the normal and shear component of the magnetic field, respectively, and Büchner et al. [1991] investigated the consequences for the collisionless tearing mode instability. However, it has to be noted that in this type of magnetic configuration, there is a gradient of the magnetic field. Curvature of the magnetic field line plays a role in particle orbits but the gradient of the magnetic field magnitude has also a strong influence as the Larmor radius directly depends on it. These two effects cannot be decorelated in such magnetic topology. One of the aim of this study is to provide some insights in the relative importance of the finite size of the reversal layer and curvature effects for particle dynamics.

Figure 7 presents the ratio between final to initial magnetic moment of particles, after one interaction with the modified TD, depending on their initial pitch angle. Initial conditions are $P_{x}=0$ and $\delta=0.01$. When $\kappa_{R L}$ 

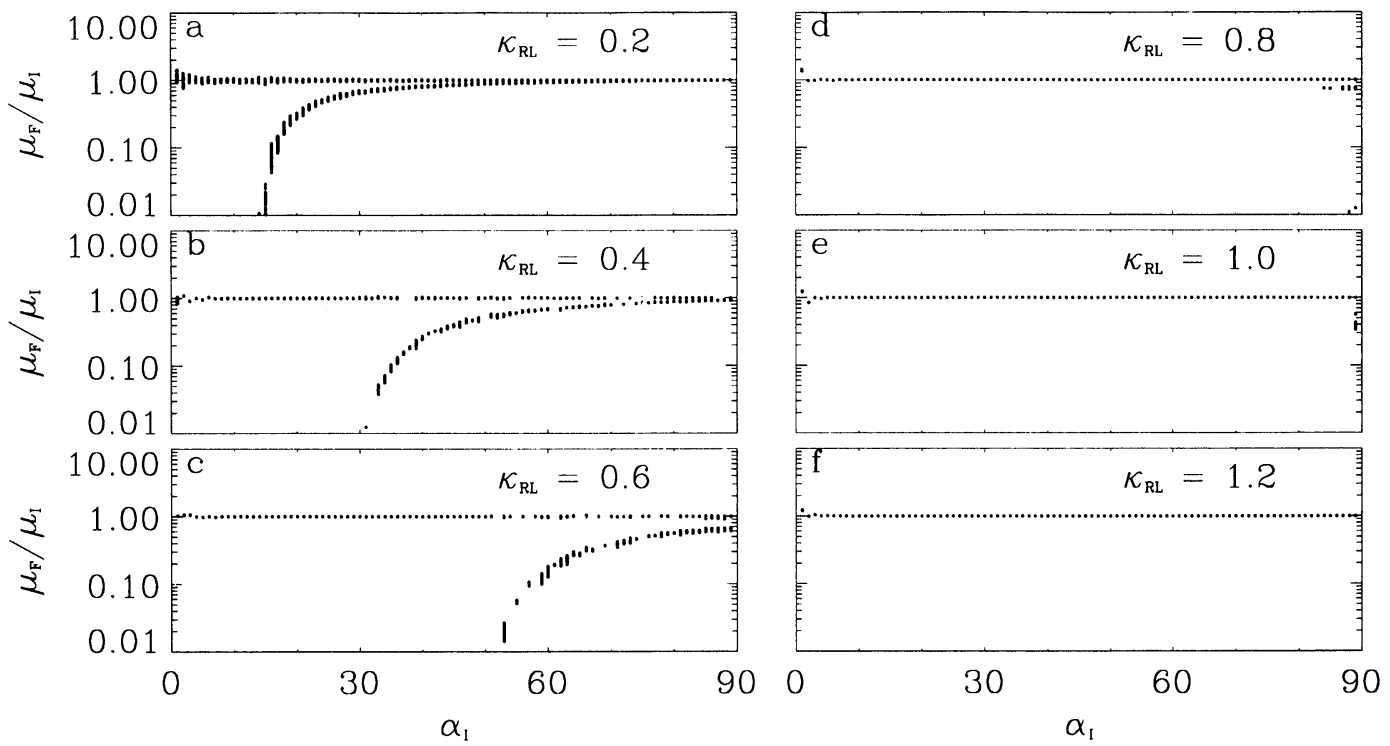

Figure 7. Ratio between final and initial magnetic moment after one interaction with the modified tangential discontinuity, depending on the initial pitch angle: (a-f) $\kappa_{S}=0.2,0.4,0.6$, $0.8,1.0$, and 1.2 , respectively.

is smaller than $0.3, \kappa_{B Z}$ is smaller than 3 . As a result, both thickness of the reversal layer and curvature effects exist in this region. Conversely, when $\kappa_{R L}$ is larger than $0.3, \kappa_{B Z}$ is larger than 3. Sergeev et al. [1983] demonstrated that above the limit $\kappa_{B Z}>3$, there is no magnetic moment diffusion. The one observed in Figure 7 is only due to the finite size of the reversal layer. Figures 7 a to $7 \mathrm{f}$ correspond to $\kappa_{R L}=0.2,0.4$, $0.6,0.8,1.0$, and 1.2 , respectively. The interval $0^{\circ}-90^{\circ}$ of pitch angle and $0^{\circ}-360^{\circ}$ of gyrophase are sampled with a $1^{\circ}$ step. This display is similar to the one used by Delcourt et al. [1996] in their Figure 1 that they called the three branch pattern. Their study was conducted in a modified HS, for which $\kappa_{B Z}$ is the parameter allowing to organize the different orbits. Figure 7 displays a horizontal branch at all pitch angle values, for which the magnetic moment is nearly constant and a skewed branch at higher pitch angle values, starting at a critical value $\alpha_{C}$, for which the magnetic moment is damped. The position of the skewed branch drifts toward larger pitch angles when increasing the $\kappa_{R L}$ value.

A major difference from the three branch pattern of Delcourt et al. [1996], is that for a given value of the pitch angle, the magnetic moment is either conserved or damped for $\alpha>\alpha_{C}$. However, the magnetic moment ratio can only reaches one of these two values, whereas the whole range of values between the limit can be reached in the three-branch pattern. In a modified HS topology, particle dynamics is chaotic, and the magnetic moment diffusion is strongly controlled by the particle's gyrophase. In the modified TD case the development of chaos is constrained, and the abscence of gradient in the total magnetic field magnitude is suspected to play a major role. Evolution of the particle magnetic moment is stochastic as after one interaction with the modified TD, the magnetic moment can be cither damped or unchanged, this value strongly depending on the initial gyrophase of the particle. However, there is a kind of determinism in this scattering process as there exist only two possible values for the final magnetic moment, assuming that the initial pitch angle is larger than $\alpha_{C}$. This is not the case in the modified HS as the whole range of magnetic moment bounded by the limits in the three branch pattern can be reached. The nature of the chaotic scattering is essentially different from the one in the modified TD. This is the meaning of "weakly chaotic."

Focusing on the particles' initial pitch angle $\alpha$, it can be seen in Figure 7 that at small $\alpha$ values, particles are not scattered when crossing the modified TD. When increasing $\alpha$, the damping occurs at the given value $\alpha_{C}$ ( $\alpha_{C}$ being a function of $\left.\kappa_{R L}\right)$, the importance of the damping decreasing when $\alpha$ increases. As $\kappa_{R L}$ increases, $\alpha_{C}$ increases and nearly vanishes at $\kappa_{R L}=1$. The resulting orbits (not presented here) show that $\kappa_{R L}=1$ is the limit between transient orbits $\left(\right.$ at $\kappa_{R L}<1$ ) like the one presented in Figure 3, experiencing both adiabatic and super-adiabatic sequences, and purely adiabatic sequences (at $\kappa_{R L}>1$ ). The population obtained after one interaction with the interface results in a mixing of initially low $\alpha$ value particles that are not scattered and high $\alpha$ value particles that are either damped or not damped during the interaction, depending on their initial gyrophase. It has to be noted that the gaps in each branch just depends on the pitch angle sampling, and could be filled with a better sampling. However a $1^{\circ}$ step provides enough accuracy to get the form of the two branches. In the same way, a 


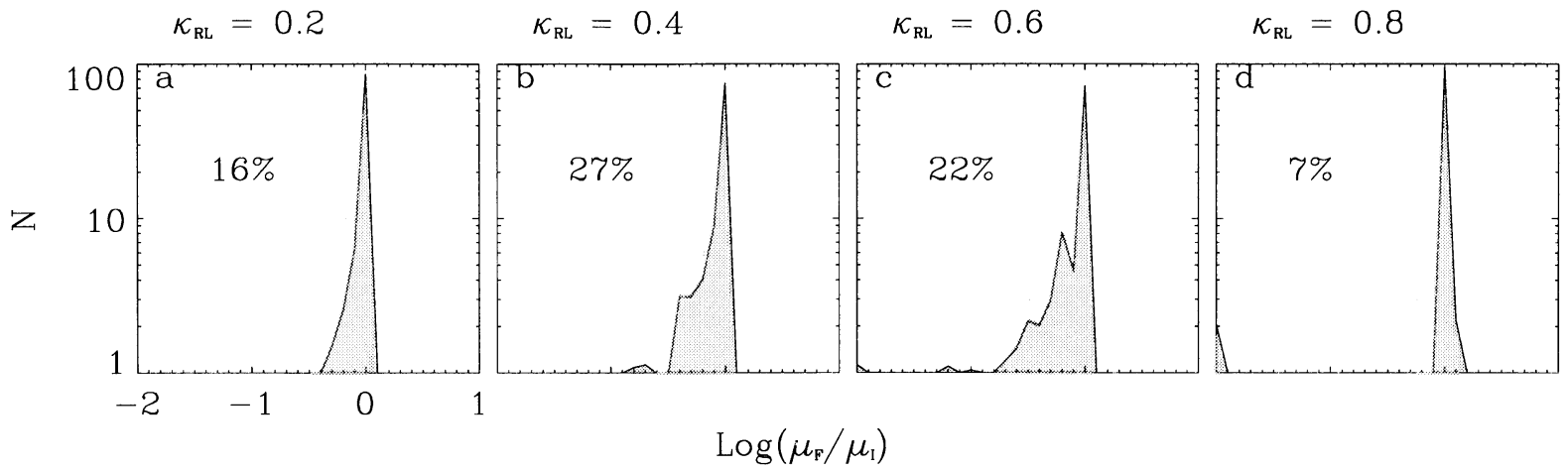

Figure 8. Magnetic moment (normalized to its initial value) after one interaction with a modified TD, depending on the $\kappa_{R L}$ value.

better sampling in gyrophase does not modify the display as all the points for a given pitch angle are almost superposed in one of the branch.

Analyzing single particle orbits associated to each branch, a clear structure appears. Orbits like the one presented in Figure $3 \mathrm{a}$ are the one associated to the skewed branch. It can be seen that the particles stay in the same side of the modified TD before and after interacting with it. On the other hand, orbits like the one presented in Figure 3c escape from the modified TD on the other side than it entered it and are the one associated to the horizontal branch. Crossing particles will have a constant $\mu$ when interacting with the modified TD, whereas noncrossing particles will have a damped magnetic moment.

Figure 8 is rather similar to Figure 7 but using a different display. It depicts the amount of particles (in percentage) scattered after one interaction with the modified TD, depending on the logarithmic value of the ratio between final to initial magnetic moment. Figures $8 \mathrm{a}$ to $8 \mathrm{~d}$ correspond to $\kappa_{R L}=0.2,0.4,0.6$, and 0.8 , respectively. The percentage in each windows correspond to the total amount of damped particles, whatever the value of the damping. It appears that for small values of $\kappa_{R L}, \sim 1 / 5$ to $1 / 4$ of the total initial population are damped when interacting with the modified TD.

Once could think that as the damped population is the one that does not cross the modified TD, no fieldaligned population can be produced at the traversal of the modified TD. However focusing on a given value of $\kappa_{R L}$, all the small pitch angle particles will cross the modified TD without being damped, whereas just a fraction will cross at larger pitch angle (still in an undamped way) the other fraction being damped but noncrossing. This process is equivalent to a high pitch angle values filtering, resulting hence in a field aligned population. A qualitative description of this pitch angle distribution as the one associated to noncrossing particles is beyond the scope of this paper. The production of a field-aligned population is consistent with the observations of hot ions streaming, collimated along the magnetic field when crossing the magnetopause during flux transfer events [see, e.g., Scholer et al., 1982].

\section{Summary and Discussion}

Because of its thickness, finite Larmor radius effects exist at the magnetopause. It is demonstrated that in a pure TD, when the IMF is southward, particle dynamics is regular. The addition of a small component of the magnetic field, normal to the interface (in the $y$ direction), breaks down the regularity of motion resulting in a weakly stochastic dynamics. This is analogous to tightly curved magnetic field geometries, where the phase space can be structured with the $\kappa_{B Z}$ parameter, defined by Büchner and Zelenyi [1989]. A new parameter, $\kappa_{R L}=\left[L_{R L} / \rho_{L \max }\right]^{1 / 2}$ (where $L_{R L}$ is the finite size of the magnetic field reversal layer) is proposed to fill the corresponding role in magnetic topology with a thin reversal layer. It is shown that particle motion is adiabatic for $\kappa_{R L}>1$ and experience transient orbits for $\kappa_{R L}<1$. Their magnetic moment is conserved at low pitch angle values, whereas it is damped at higher pitch angle values, leading to the production of a field-aligned population when crossing the magnetopause.

Other calculations, similar to those presented in Figure 7 and 8 , have been carried out for different values of $\delta$. We focused on the results obtained for $\kappa_{B Z}>3$ to avoid all the curvature effects. In this way, the obtained magnetic moment damping can only be due to the finite size of the reversal layer. For whatever values of $\delta$, smaller or larger than $\delta=0.01$ as in Figure 7 and 8 , the noncrossing particles are pitch angle damped when interacting with the modified TD at $\kappa_{R L}<1$. The efficiency of this damping may slightly depend on the $\delta$ value, but the important point is that $\delta$ is not a critical parameter. It does not control the existence of the pitch angle damping and more generally the existence of the two branch pattern at low $\kappa_{R L}$ value. Furthermore, the calcluations performed with different $\delta$ values show that $\kappa_{R L}$ is unchanged, the obtained results are somewhat similar displaying also a two-branch pattern, 
whereas $\kappa_{B Z}$ depends on $\delta$. By the way, $\kappa_{B Z}$ cannot be used as an adequate parameter in such a magnetic topology as it depends on $\delta$.

On the other hand, results are different if we consider different values for the polarization of the TD. The anisotropy, obtained after traversal of the magnetopause, disappears if we consider a rotation of $90^{\circ}$ (IMF in the $-X$ direction). The magnitude of the magnetic shear is a controlling parameter for the flow direction resulting on the magnetopause traversal. A parametric study would be necessary to investigate the pitch angle distribution resulting from interaction with a modified TD at different magnetic shears. However, computation with a rotation of the magnetic field through the discontinuity of $175^{\circ}, 170^{\circ}$, and $165^{\circ}$ have been performed and a similar two-branch pattern has been obtained. 'The results obtained at $180^{\circ}$ are not a singularity of the model. Pitch angle scattering obtained for different values of the magnetopause polarization could explain the production of bulk flow in particular directions [see, e.g., Eastman and Frank, 1982]. Another point to be raised is that the study presented in this paper is valid when $\delta=b_{n} / b_{0}$ is small, meaning that the discontinuity is essentially tangential. We suspect the results to be strongly modified if $\delta$ becomes close to or larger than one, as it can be the case for rotational discontinuity.

The results of this study can thus be summarized in the following four points.

1. Charged particle dynamics is regular in a tangential discontinuity and a constant of motion can be defined. This type of magnetic boundary does not allow any mass exchange.

2 . In the same topology the addition of a small component to the magnetic field, normal to the interface, imply the loss of a constant of the motion and introduce a weakly stochastic behavior.

3. A new parameter $\kappa_{R L}=\left[L_{R L} / \rho_{\text {Lmax }}\right]^{1 / 2}$ (where $L_{R L}$ is the size of the reversal layer) is proposed to organize the different possible orbits. When $\kappa_{R L}>1$, particle motion is regular, and the magnetic moment $\mu=m V_{\perp} / q b$ is conserved. When $\kappa_{R L}<1$, particle motion is weakly stochastic, and the magnetic moment is either conserved or damped, depending on the initial pitch angle and gyrophase.

4. The existence of a small component of the magnetic field, normal to the magnetopause, allows the particles to cross the magnetopause. In the southward IMF case, with a small component of the magnetic field normal to the magnetopause, particles are pitchangle scattered when crossing the magnetopause and the resulting population is mainly field aligned.

The existence of a small magnetic field component normal to the interface means that the magnetic field lines from magnetosphere and magnetosheath are connected. This results in breaking down the regularity of particle dynamics but implies the existence of a re- connection process which origin is still not elluded in collisionless plasma. For the Earth magnetopause the development of a Kelvin-Helmholtz instability or the existence of electrostatic turbulence could also lead to the existence of small scale lengths and modify the magnetic field topology. This would allow transition between adiabatic and super-adiabatic motion, giving rise to chaotic motions and traversal across the magnetopause. The efficiency of this process could also provide for mass exchanges at the magnetopause.

Acknowledgments. The author thanks Dominique C. Delcourt and Thomas E. Moore for useful discussions and suggestions. This work was performed while the author held a National Research Council-NASA/GSFC Resident Research Associateship.

Janet G. Luhmann thanks Lev Zelenyi and Richard F. Martin Jr. for their assistance in evaluating this paper.

\section{References}

Berchem, J., and C. T. Russell, The thickness of the magnetopause current layer: ISEE 1 and 2 observations, $J$. Geophys. Res., 87, 2108, 1982a.

Berchem, J., and C. T. Russell, Magnetic field rotation through the magnetopause: ISEE 1 and 2 observations, J. Geophys. Res., 87, 8139, 1982b.

Büchner, J., and L. M. Zelenyi, Regular and chaotic charged particle motion in magnetotaillike field reversals 1. Basic theory of trapped motion, J. Geophys. Res., 9/4, 11,821, 1989.

Büchner, J., and L. M. Zelenyi, The separatrix tentacle effect of ion acceleration to the plasma sheet boundary, Geophys. Res. Lett., 17, 127, 1990.

Büchner, J., and L. M. Zelenyi, Regular and chaotic particle motion in sheared magnetic field reversals, Adv. Space Res., 11, 177, 1991 .

Büchner, J., M. Kuznetsova, and L. M. Zelenyi, Sheared field tearing mode instability and creation of flux ropes in the Earth magnetotail, Geophys. Res. Lett., 18, 385, 1991.

Burkhart, G. R., P. B. Dusenbery, and T. W. Speiser, Particle chaos and pitch angle scattering, J. Geophys. Res., 100, 107, 1995.

Chen, J., Nonlinear dynamics of charged particles in the magnetotail, J. Geophys. Res., 97, 15,011, 1992.

Chen, J., and P. J. Palmadesso, Chaos and nonlinear dynamics of single particle orbits in a magnetotaillike magnetic field, J. Geophys. Res., 91, 1499, 1986.

Curran, D. B., and C. K. Goertz, Particle distributions in a two-dimensional reconnection field geometry, J. Geophys. Res., 94, 272, 1989.

Curran, D. B., C. K. Goertz, and T. A. Whelan, Ion distributions in a two-dimensional reconnection field geometry, Geophys. Res. Lett., 14, 99, 1987.

Delcourt, D. C., R. F. Martin Jr., and F. Alem, A simple model of magnetic moment scattering in a field reversal, Geophys. Res. Lett., 21, 1543, 1994.

Delcourt, D. C., J.-A. Sauvaud, R. F. Martin Jr., and T. E. Moore, On the nonadiabatic precipitation of ions from the near-Earth plasma sheet, J. Geophys. Res., 101, 17,409, 1996.

Eastman, T. E., and L. A. Frank, Observations of high-speed plasma flow near the Earth's magnetopause: Evidence for reconnection?, J. Geophys. Res., 87, 2187, 1982.

Eckhardt, B., Irregular scattering, Physica D, 33, 89, 1988. 
Harris, E. G., On a plsama sheath separating regions of oppositely directed magnetic field, Nuovo Cimento, 23, $115,1962$.

Holland, D. L., J. Chen, and A. Agranov, Effect of a constant cross-tail magnetic field on the particle dynamics in the magnetotail, J. Geophys. Res., 101, 24,997, 1996.

Karimabadi, H., P. L. Pritchett, and F. V. Coroniti, Particle orbits in two-dimensional equilibrium models for the magnetotail, J. Geophys. Res., 95, 17,153, 1990.

Landau, L. D., and E. M. Lifshitz, Mechanics, AddisonWesley, Reading, Mass., 1960.

Le, G., and C. T. Russell, 'The thickness and structure of high beta magnetopause current layer, Geophys. Res. Lett., 21, 2451, 1994 .

Lichtenberg, A. J., and M. A. Liebermann, Regular and Chaotic Dynamics, Appl. Math. Sci., vol 38, SpringerVerlag, New York, 1983.

Martin, R. F., Jr., T. W. Speiser, and K. Klamczynski, Effect of $B_{y}$ on neutral line ridges and dynamical source ordering, J. Geophys. Res., 99, 23,623, 1994.

Neff, J. E., T. W. Speiser, and D. J. Williams, Magnetosheath quasi-trapped distributions and ion flows associated with reconnection, J. Geophys. Res., 92, 1177, 1987.

Papamastorakis, I., G. Paschmann, N. Sckopke, S. J. Bame, and J. Berchem, The magnetopause as a tangential discontinuity for large field rotation angles, J. Geophys. Res., 89, 127, 1984.

Scholer, M., D. Hovestadt, F. M. Ipavich, and G. Glockler, Energetic protons, alpha particles, and electrons in magnetic flux transfer events, J. Geophys. Res., 87, 2169, 1982.

Sergeev, V. A., E. M. Sazhina, N. A. Tsyganenko, J. A. Lundblad, and F. Soras, Pitch-angle scattering of ener- getic protons in the magnetotail current sheet as the dominant source of their isotropic precipitation into the nightside ionosphere, Planet Space Sci., 31, 1147, 1983.

Sonnerup, B. U. O., Adiabatic particle orbits in a magnetic null sheet, J. Geophys. Res., 76, 8211, 1971.

Sonnerup, B. U. Ö., and L. J. Cahill, Magnetopause structure and attitude from Explorer 12 observations, J. Geophys. Res., 72, 171, 1967.

Speiser T. W., Particle trajectory in model current sheets, 1, Analytical solutions, J. Geophys. Res., 70, 4219, 1965.

Speiser T. W., Conductivity without collisions or noise, Planet. Space Sci., 18, 613, 1970.

Speiser, T. W., D. J. Williams, and H. A. Garcia, Magnetospherically trapped ions as a source of magnetosheath energetic ions, J. Geophys. Res., 86, 723, 1981.

Swift, D. W., and L. C. Lee, Rotational discontinuity and the structure of the magnetopause, J. Geophys. Res., 88, $111,1983$.

Williams, D. J., and T. W. Speiser, Sources for energetic ions at the plasmasheet boundary: Time varying or steady state?, J. Geophys. Res., 89, 8877, 1984.

Zhu, Z., and G. Parks, Particle orbits model current sheet with a nonzero $B_{y}$ component, J. Geophys. Res., 98, 7603, 1993.

R. Smets, Centre d'étude des Environnements Terrestre et Planétaires, Centre National de la Recherche Scientifique, 78140 Vélizy, France.

(Received May 18, 1999; revised May 15, 2000; accepted June 5, 2000.) 